\title{
Broadband impulsive vibrational spectroscopy of excited electronic states in the time domain
}

\author{
Matz Liebel and Philipp Kukura*
}

Physical and Theoretical Chemistry Laboratory, Department of Chemistry, University of Oxford, South Parks Road, Oxford OX1 3DP

*philipp.kukura@chem.ox.ac.uk 
Ultrafast vibrational spectroscopy is a powerful tool for studies of chemical reaction dynamics. Here, we introduce broadband impulsive vibrational spectroscopy and demonstrate that it is capable of recording full vibrational spectra of excited electronic states in the time-domain. The resulting spectra do not suffer from the non-trivial baselines and lineshapes often encountered in frequency-domain techniques and enable optimal subtraction of background signatures. Probing the molecular dynamics continuously over a broad energy bandwidth makes it possible to assign the observed coherences to specific electronic states and suggests the existence of mode specific absorption spectra reminiscent of resonance Raman intensity analysis. The first observation of the nominally forbidden one-photon ground to first excited electronic state transition in $\beta$ carotene demonstrates the high sensitivity of our approach. Our results provide a first glimpse of the immense potential of broadband impulsive Raman spectroscopy to study ultrafast chemical reaction dynamics.

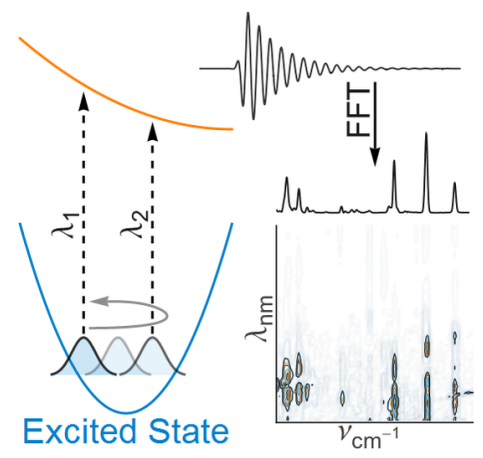

Table of Contents Figure

Keywords: Ultrafast spectroscopy, time-domain vibrational spectroscopy, vibrational coherence, carotenoids, femtosecond Raman 
Our understanding of chemical reactivity is fundamentally linked to the nature and timing of atomic and electronic motion. Observing the changes in molecular structure during a chemical reaction is thus one of the most direct ways to study chemical reaction dynamics. This notion of recording a molecular movie has motivated the development of a series of sophisticated experimental techniques over the past decades. ${ }^{1-3}$ In terms of combining high structural sensitivity with excellent temporal resolution, vibrational spectroscopies have been particularly successful, ${ }^{4,5}$ but a number of fundamental limitations remain. Femtosecond infrared (IR) spectroscopy requires tunable sources in the IR, where the achievable energy bandwidths and pulse durations are limited. Previous knowledge of the spectral region of interest is therefore required and the achievable time-resolution is poor compared to electronic techniques operating in the visible region of the electromagnetic spectrum. Frequency-domain coherent Raman spectroscopies, such as picosecond time-resolved resonance Raman $\left(\mathrm{TR}^{3}\right)$, coherent anti-Stokes Raman (CARS) and femtosecond stimulated Raman (FSRS) are capable of recording full vibrational spectra with a time-resolution comparable to the vibrational dephasing time..$^{4,6,7}$ With respect to the initiation of vibrational coherence, both CARS and FSRS can achieve temporal accuracies of well below 100 fs and thereby access structural dynamics that occur on even faster timescales. ${ }^{8,9}$ All of these techniques, however, suffer from interfering lineshapes and non-trivial backgrounds especially when tuned into resonance with an electronic transition making it difficult to take advantage of resonance enhancement. In addition, the experimental results are necessarily averaged over the vibrational dephasing time preventing direct access to the underlying coherent nuclear motion. ${ }^{10,11}$

Impulsive stimulated Raman spectroscopy (ISRS) is a time-domain alternative for recording Raman activity. ${ }^{12-14}$ A short pulse, whose bandwidth exceeds the vibrational energy spacing, 
creates a nuclear wavepacket that evolves as a function of time. The resulting non-stationary state can be probed by recording oscillatory changes of the refractive index or electronic spectra at the frequency of the impulsively excited oscillation. This approach has been successful in revealing nuclear coherence on ground electronic states in agreement with frequency domain techniques such as continuous wave resonance Raman spectroscopy but at the cost of vastly increased experimental complexity. ${ }^{15-17}$ Extending ISRS studies to excited electronic states, however, has been elusive. This is partially a consequence of the difficulty associated with the observation of high-frequency coherence with vibrational periods as short as $10 \mathrm{fs}$ in the time domain, but is also caused by the unavoidable presence of ground state and solvent coherences that are often orders of magnitude larger. As a consequence, the observation of vibrational coherence on excited electronic states has either been restricted to low-frequency modes,${ }^{18,19}$ or required experimentally more demanding approaches, such as degenerate four wave mixing $(\mathrm{DFWM}) .^{20,21}$

Here, we introduce broadband impulsive vibrational spectroscopy (BB-IVS) and show that it is capable of revealing vibrational coherence in the time-domain over the full vibrational energy window of interest $\left(0-2000 \mathrm{~cm}^{-1}\right)$. BB-IVS has three fundamental advantages over current stateof-the-art frequency and time-domain techniques: (1) it requires only one moderately short $(\sim 12$ fs) optical pulse resulting in a comparatively simple experimental setup. (2) Vibrational coherence can be probed over a large energy window allowing for confident assignment of the observed wavepacket activity to specific electronic states. (3) Recording vibrational coherence in the time-domain makes it possible to use an automated background subtraction procedure that avoids artefacts and bias commonly encountered in the frequency domain. For this initial report, 
we use $\beta$-carotene as a model system because it is has been studied extensively ${ }^{17,22,23}$ and represents a benchmark for direct comparison with the state-of-the-art.

Optical excitation of $\beta$-carotene $(<500 \mathrm{~nm})$ populates the second excited singlet state, $\mathrm{S}_{2}$, that decays quickly ( 200 fs) to the first excited state, $S_{1}$ (Figure $\left.\left.1 \mathrm{~A}\right)\right)^{24,25}$ Direct, one-photon excitation to $S_{1}$ is forbidden by symmetry. The $S_{1}$ state exhibits a characteristic absorption band centred at $560 \mathrm{~nm}$ (Figure 1B) that decays with a 8 ps time constant as the system relaxes back to the ground electronic state. In our experiment, a $200 \mathrm{fs}$ excitation pulse resonant with the $\mathrm{S}_{2} \leftarrow \mathrm{S}_{0}$ transition generates a mixture of molecules in the $S_{0}$ and $S_{1}$ electronic states in the probed region. After a time-delay of $2 \mathrm{ps}$, we then probe this mixture using broadband impulsive vibrational spectroscopy with a short (12 fs) impulsive and a time-delayed, chirped white light probe pulse (Figures 1C, D).

Our approach is similar to the seminal work by Tahara and coworkers ${ }^{18}$ but differs in the detection methodology. As a consequence, we only require one short impulsive pulse and probe the subsequent dynamics with a readily available chirped white light continuum of $\sim 300$ fs duration making the detection ultrabroadband and thereby covering multiple absorption and emission bands. In addition, using a white-light probe pulse minimises the laser noise in the detection channel ${ }^{26}$ and enables spectroscopic sensitivities down to the $10 \mu \mathrm{OD}$ level. ${ }^{27,28}$ Such a pump-probe arrangement is still capable of resolving high-frequency oscillations for modes up to $3000 \mathrm{~cm}^{-1}$, corresponding to a vibrational period of only $11 \mathrm{fs}$, even though the nominal temporal resolution of the experiment is $\sim 300$ fs. The key is to achieve sufficient spectral resolution and energy bandwidth in the detection, such that the temporal chirp enables a time-resolution at the theoretical limit of a transform limited probe pulse, that can be $<3$ fs for a white light continuum..$^{29-31}$ In our case, we have found a moderate energy resolution of $100 \mathrm{~cm}^{-1}$ for probe 
wavelengths ranging from 500 - $900 \mathrm{~nm}$ sufficient for a 12 fs pump pulse and a commonly chirped white light probe..$^{27}$

With such an approach, we observe high-frequency vibrational coherence along the full bandwidth of our detection window $(510-780 \mathrm{~nm})$ as shown in the transient differential absorbance map for neat acetonitrile (Figure 2A). Coherent oscillations with a period on the order of $\sim 35$ fs are visible throughout the entire probed region despite the long duration of the probe pulse. A slice along $750 \mathrm{~nm}$ exhibits a coherent artefact around time zero ${ }^{32}$ followed by a single visible oscillation (Figure 2B). Fourier transformation of the oscillatory part reveals additional oscillatory components and generates the well known Raman spectrum of acetonitrile, albeit with a changed intensity pattern. This is partly caused by the limited temporal resolution ( $<15 \mathrm{fs}$ ) of our experiment, making the observation of the highest frequency oscillations with periods as short as $11 \mathrm{fs}\left(3000 \mathrm{~cm}^{-1}\right)$ extremely challenging. Nevertheless, we can observe the C$\mathrm{H}$ stretch at $2941 \mathrm{~cm}^{-1}$ throughout our detection window as shown in the inset. The lower magnitude of the $\mathrm{C}-\mathrm{H}$ stretch in the NIR compared to the visible is caused by group velocity mismatch in the sample between the impulsive pump and the probe wavelength (inset).

Having shown that our impulsive-pump/chirped-probe experiment is capable of revealing coherent nuclear motion over the full vibrational frequency window, we now turn to the first excited electronic state of $\beta$-carotene. We repeat the experiment shown in Figure $2 \mathrm{~A}$ on $\beta$ carotene dissolved in cyclohexane but with an additional excitation pulse (Figure 1C). The corresponding differential absorbance map can be thought of as a standard transient absorption experiment, but this time on the $S_{1}$ state, because the impulsive pulse is only resonant with the $\mathrm{S}_{\mathrm{y}} \leftarrow \mathrm{S}_{1}$ transition. The impulsive pulse removes $\mathrm{S}_{1}$ population and thereby causes an increase in the probe transmission at $560 \mathrm{~nm}$ that recovers partially with a $210 \mathrm{fs}$ time constant (Figure 2C). 
We remark that the signal modulations evidenced by the contour maps are entirely reproducible as they are caused by nuclear wavepacket motion. A slice along the peak of the $\mathrm{S}_{\mathrm{y}} \leftarrow \mathrm{S}_{1}$ absorption reveals small oscillations on top of the electronic signal (Figure 2D), with the Fourier power spectrum resembling a mixture of the Raman peaks of cyclohexane $\left(803 \mathrm{~cm}^{-1}\right)$, the $\mathrm{S}_{0}$ $\left(1532 \mathrm{~cm}^{-1}\right)$ and the $\mathrm{S}_{1}$ state $\left(1798 \mathrm{~cm}^{-1}\right)$ of $\beta$-carotene.

The large magnitude of both solvent and ground state $\beta$-carotene bands calls for an efficient subtraction methodology to isolate the desired vibrational coherence and thus spectrum of the $S_{1}$ state. The subtraction is usually performed by manually adjusting subtraction coefficients of independently recorded spectra. Finding the correct coefficients is a highly subjective process, especially in the often encountered case of overlapping ground and excited state features. In addition, subtraction in the frequency domain necessarily induces artefacts in the form of dispersive features that reduce the quality of the final spectra and are almost invariably observed in time-resolved spontaneous and coherent Raman spectra. ${ }^{22,23}$ To illustrate this effect, we have simulated the coherent response from a mixture of a solvent (340 and $\left.410 \mathrm{~cm}^{-1}\right)$ and solute (450 and $600 \mathrm{~cm}^{-1}$ ), choosing only two frequencies for each species for convenience (Figure 3A). The corresponding Fourier transform exhibits four bands at the respective positions, linewidths and amplitudes of the original coherences. Fourier transforming the coherence prior to the subtraction, a process that is unavoidable in frequency-domain techniques, necessarily results in dispersive features at the positions of the solvent bands because of the separation of phase and amplitude in Fourier spectra. If the subtraction is performed in the time-domain, however, these features can be completely avoided (Figure 3B).

The ability to switch between the time and frequency domains makes it possible to use an iterative algorithm to optimally subtract ground state coherences from our spectra (Figure 3C). 
We begin with the measured solvent-only (SOC) and solvent-plus-solute coherences (SPSC) and apply a Kaiser window to suppress side-lobes in the Fourier transform (i). The windowed coherences are then fed into an iterative algorithm that uses two variables: the amplitude $A$ and a phase shift $\Delta \phi$ to scale and shift the solvent coherence. Variation of these parameters is necessary because of unavoidable changes in pulse energies and time delays for different experiments. The resulting SOC is then subtracted from the SPSC (ii) resulting in a first guess for the solute-only coherence (iii). To evaluate the fidelity of the first guess of $A$ and $\Delta \phi$, we perform a Fourier transform that resolves all present frequencies. Evaluating the real and imaginary parts of the Fourier transform makes it possible to obtain gradients for both the time shift (phase) and the amplitude (iii). A Levenberg-Marquardt algorithm then computes the gradients and generates a new guess for $A$ and $\Delta \phi$ that is applied in the second iteration (ii). This procedure is repeated until a pre-determined convergence criterion is reached (iv). We evaluate the degree of convergence in a limited frequency window (grey box) ideally containing isolated ground state modes. This joint time and frequency-domain approach has the fundamental advantage that it allows for optimization in the frequency domain, where solvent and solute oscillations can be well separated, but performs the subtraction in the time domain, which enables the generation of an artefact-free final spectrum. The subtraction of ground state coherences in the time domain alone can in principle be optimised as shown in Figure 3A, but has the disadvantage that one loses the molecule-specific gradient that can be exploited by selecting specific spectral regions of interest.

Repeating the presented process of analysing individual wavelengths, subtracting broad electronic kinetics, windowing and eventually generating Fourier power spectra over the entire wavelength range of our probe window generates Fourier maps as a function of wavelength 
(Figure 4A). Prior to any subtraction, the intense cyclohexane ring breathing mode at $803 \mathrm{~cm}^{-1}$ dominates the appearance of the map, but a number of peaks in the $\mathrm{C}-\mathrm{C}$ and $\mathrm{C}=\mathrm{C}$ stretching region from $1100-1600 \mathrm{~cm}^{-1}$ are also visible. Averaging over the visible region, shaded in Figure 4A, generates the spectrum in Figure 4B. Subtraction of the solvent signatures, which are recorded independently in the absence of $\beta$-carotene, removes the most dominant feature at 803 $\mathrm{cm}^{-1}$ and low frequency, $\mathrm{C}-\mathrm{C}$ and $\mathrm{C}=\mathrm{C}$ stretching modes dominate the Fourier map (Figure 4C). The frequency spectrum in Figure 4D exhibits $S_{0}$ peaks at 1027,1160 and $1532 \mathrm{~cm}^{-1}$, and first signs of known $\mathrm{S}_{1}$ features become visible, such as the $\mathrm{C}-\mathrm{C}$ stretch at $1256 \mathrm{~cm}^{-1}$ and the $\mathrm{C}=\mathrm{C}$ band at $1798 \mathrm{~cm}^{-1}$. Subtraction of ground state features then reveals an $\mathrm{S}_{1}$-only map of vibrational coherence (Figure 4E). The Fourier intensity is now exclusively focussed on the $500-650 \mathrm{~nm}$ region, as expected from the $\mathrm{S}_{1} \leftarrow \mathrm{S}_{\mathrm{y}}$ absorption band (Figure 1B). The wavelength averaged spectrum exhibits the highly upshifted $\mathrm{C}=\mathrm{C}$ band at $1798 \mathrm{~cm}^{-1}$, another at $1540 \mathrm{~cm}^{-1}$ and a C-C stretch at $1256 \mathrm{~cm}^{-1}$ (Figure 4F). These features are in excellent agreement, both in position and intensity with previous ps-resonance Raman results on the $S_{1}$ state of $\beta$-carotene, ${ }^{22}$ but show some discrepancy with recent FSRS results, most likely due to the difficulties associated with the ground state and baseline subtraction process. ${ }^{23}$ The strong Raman activity in the low frequency region has previously not been observed due to the difficulty of accessing this region in frequency-domain Raman.

The Fourier maps in Figure 4 exhibit a clear wavelength dependence of the Fourier intensities for all of the observed vibrational modes. The well established relationship between the shape of the electronic resonance and the magnitude of vibrational oscillations ${ }^{16}$ suggests that we are observing mode-specific absorption spectra. This feature is reminiscent of resonance Raman intensity analysis ${ }^{33}$ and will be subject of future investigations. Overall, the wavelength- 
dependence of the observed oscillatory amplitudes allows for a confident assignment of the probed coherence to specific electronic states.

The experimental data presented in Figure 4 suggest that our approach is capable of generating broad vibrational spectra of excited electronic states of excellent quality. Not only can we access the low frequency region of the spectrum, but even weak Raman transitions are resolvable with high sensitivity (inset of Figure 5A). The two bands at 785 and $813 \mathrm{~cm}^{-1}$ are 1000-times smaller than the original solvent band at $803 \mathrm{~cm}^{-1}$, but are nevertheless resolvable with high sensitivity. We have confirmed that these bands are indeed representative of vibrational coherence on the $S_{1}$ state by repeating the experiment in deuterated chloroform that does not show any Raman activity in this region (dashed line). We also emphasize that all the presented spectra are generated from the raw data without any additional smoothing or fitting. We only applied a Kaiser-Bessel window followed by apodisation to suppress the formation of large side lobes and avoid pixelation in the Fourier power spectra, respectively.

In the experiments discussed so far, we used an additional excitation pulse to generate significant $S_{1}$ population in the sample because the impulsive pulse is non-resonant with the $\mathrm{S}_{2} \leftarrow \mathrm{S}_{0}$ transition (see Figure 1). In the absence of the excitation pulse, we should only observe vibrational signatures due to ground state $\beta$-carotene and the solvent (Figure 5B) because no population in $S_{1}$ is present when the impulsive pulse interacts with the sample. Interestingly, even in the absence of the UV excitation pulse, we observe the most prominent vibrational feature of the $S_{1}$ state of $\beta$-carotene, the $C=C$ stretch $1798 \mathrm{~cm}^{-1}$, but with almost vanishingly small intensity (inset). This is a priori puzzling: the impulsive pump may cause electronic excitation, but only into the $S_{2}$ state, which decays with a 200 fs time-constant to $S_{1}$. A tempting interpretation would be that the short pulse generates a nuclear wavepacket in the $\mathrm{C}=\mathrm{C}$ mode in 
$\mathrm{S}_{2}$ which survives the surface crossing and is subsequently observed in our experiment. This is, however, not possible, because the spectral overlap between the ground state absorption spectrum and that of the impulsive pulse does not allow for superimposing two high-frequency vibrational energy levels on the $S_{2}$ state. By recording transient spectra prior to the nominal zero time-delay between impulsive and probe pulses, we can also place an upper limit on the excited state population generated by a experimentally induced pre-pulse. The resulting high-frequency peak at $1798 \mathrm{~cm}^{-1}$, however, would be three orders of magnitude smaller than what is observed experimentally. The most straightforward explanation is therefore that the formally symmetry forbidden $S_{1} \leftarrow S_{0}$ transition is actually weakly allowed. Thus, even in the absence of the excitation pulse, the impulsive pulse generates high-frequency coherence in $S_{1}$ by direct, onephoton excitation. This interpretation is strengthened by the perfect spectral overlap between our impulsive pulse and the $S_{1} \leftarrow S_{0}$ absorption band. ${ }^{34}$ Even for an integration time of only 10 min, we observe this weak transition by detection of high-frequency vibrational coherence, even though it has never been observed previously, even with electronic spectroscopy. Increasing the acquisition time to 120 min significantly improves the signal-to-noise ratio towards levels that are comparable with state-of-the-art frequency domain techniques, albeit measured for an extremely weak transition.

An immediate question that arises from these results is why high-frequency vibrational coherence in excited electronic states has been so difficult to observe previously. This is partially due to the strong dependence of the oscillatory amplitude of high-frequency oscillations on the available temporal resolution. In previous approaches, multiple, ultrashort pulses were required to achieve sufficient time resolution $(<15 \mathrm{fs})$, a procedure that is experimentally challenging. At the same time, Fourier power spectra dramatically enhance population effects: If $10 \%$ of the 
sample is excited, excited state features will appear 100-times smaller than those due to the ground state, making them difficult to detect. This phenomenon can be partially offset by resonance enhancement, but appears to have been insufficient to amplify excited state coherences beyond the noise level. The key to BB-IVS is that it combines high temporalresolution with high spectroscopic sensitivity and a broadband detection window. Using broadband detection ensures that the effective temporal resolution is essentially limited by the duration of the impulsive pulse, lowering the requirement for ultrashort pulse durations $(<10 \mathrm{fs})$. In fact, amplifying a portion of the white light continuum necessarily reduces the bandwidth and introduces noise. Taken together, these effects make it considerably more difficult to reveal highfrequency vibrational coherence. Because the decisive factor in determining the time-resolution for broadband detection is the transform limited pulse duration of the probe pulse, not the actually obtained duration, using the combination of an uncompressed white light continuum with a 12 fs impulsive pulse is actually preferable to the experimentally much more challenging generation and use of two $<10$ fs pulses. Ultrabroadband detection plays another, equally important, role by making it possible to confidently assign vibrational coherence to its respective electronic state. For instance, the wavelength-dependence of the vibrational signatures shown in Figure 4E allows for a direct assignment to the $S_{1}$ electronic state of $\beta$-carotene by comparison with the $\mathrm{S}_{\mathrm{y}} \leftarrow \mathrm{S}_{1}$ absorption spectrum.

With respect to frequency domain techniques, such as FSRS or CARS, BB-IVS does not suffer from non-linear backgrounds that make data analysis and interpretation difficult. We can take full advantage of resonance enhancement, one of the most useful properties of Raman spectroscopy, by deliberately probing the electronic transitions of interest without suffering from fluorescence or non-linear backgrounds. In addition to providing Raman spectra, the broadband 
detection scheme yields a clear wavelength dependence of the observed coherence and their intensities, a feature that will provide detailed information about the associated electronic potential energy surfaces and simplifies vibrational assignments. In this letter, we have not presented the temporal evolution of the excited state nuclear coherence as a function of the time delay between the excitation and impulsive pulses, because these dynamics are well known for $\beta$-carotene from previous, frequency resolved experiments. ${ }^{35}$ Reducing the duration of the excitation pulse to $<50 \mathrm{fs}$ in future experiments and recording vibrational coherence as a function of time-delay between excitation and impulsive pulses will enable studies of ultrafast vibrational structural dynamics with unprecedented sensitivity. Given the possibilities of applying well established data analysis methodologies from nuclear magnetic resonance, and the additional opportunities provided by observing vibrational coherence in the time-domain, we believe that BB-IVS has the potential of opening up completely new avenues for studies of ultrafast chemical reaction dynamics. 


\section{Experimental}

Laser pulses were delivered by a Pharos-6W amplifier $(1030 \mathrm{~nm}, 180 \mathrm{fs}, 5.5 \mathrm{~W}$ at $10 \mathrm{kHz}) .1 .5$ $\mu \mathrm{J}$ of the output generate white-light (WL) in sapphire which is used as a chirped broadband probe (300 fs, $2.5 \mathrm{~nJ}$ ) after removal of the residual fundamental. The remainder of the amplifier output is frequency doubled followed by sum frequency generation to generate $250 \mu \mathrm{J}$ at $515 \mathrm{~nm}$ and $35 \mu \mathrm{J}$ at $343 \mathrm{~nm}$. The latter pumps a two-stage WL-seeded non-collinear optical parametric amplifier (NOPA) pumped at $343 \mathrm{~nm}(35 \mu \mathrm{J})$ that generates the impulsive pump (600 nm, $7 \mu \mathrm{J})$. The pulse is compressed by chirped-mirrors (Layertec) to 12 fs verified by SHG-FROG in a 10 $\mu \mathrm{m}$ BBO crystal. A four-stage WL-seeded NOPA pumped at nm $(250 \mu \mathrm{J})$ generates narrow bandwidth pulses centred at $920 \mathrm{~nm}$ that are then frequency doubled to yield the excitation pulse (460 nm, $12 \mu \mathrm{J}, 200 \mathrm{fs}$ ). The pulse duration was estimated by the rise of the bleach signal in a $\beta$ carotene pump-probe experiment. The time delay between excitation and impulsive pulses is fixed at 2 ps and the delay of the probe pulse is varied in 3.3 fs steps with a closed-loop-piezo translation stage for a total of 2 ps with a 100 ms integration time per step. Transient absorption traces in the absence and presence of excitation and impulsive pulses are obtained by employing a double chopping scheme. A home built single shot prism spectrograph equipped with a CMOSarray detector (LW-ELIS-1024A-1394) is used for broadband detection. $\beta$-Carotene (SigmaAldrich) was used as received to prepare solutions of $\mathrm{OD}=1$ at $460 \mathrm{~nm}(200 \mu \mathrm{m})$ in cyclohexane and deuterated chloform. The sample is flowed through a cell with $200 \mu \mathrm{m}$ path length and 120 $\mu \mathrm{m}$ windows at a rate sufficient to replenish the sample between consecutive excitation pulses. The diameters of the probe, impulsive and excitation beams were $55 \mu \mathrm{m}$ and $88 \mu \mathrm{m}$ and $120 \mu \mathrm{m}$ with pulse energies adjusted to $2.5,35$ and $200 \mathrm{~nJ}$, respectively. 


\section{AUTHOR INFORMATION}

\section{Corresponding Author}

*E-mail: philipp.kukura@chem.ox.ac.uk

\section{Funding Sources}

P.K. is supported by a Career Acceleration Fellowship by the EPSRC (EP/H003541).

\section{Notes}

The authors declare no competing financial interest. 


\section{REFERENCES}

(1) Barty, A.; Küpper, J.; Chapman, H. N. Molecular Imaging Using X-Ray Free-Electron Lasers. Annu. Rev. Phys. Chem. 2013, 64, 415-435.

(2) Flannigan, D. J.; Zewail, A. H. 4D Electron Microscopy: Principles and Applications. Accounts Chem. Res. 2012, 45, 1828-1839.

(3) Sciaini, G.; Miller, R. J. D. Femtosecond electron diffraction: heralding the era of atomically resolved dynamics. Rep. Prog. Phys. 2011, 74, 096101.

(4) Hamaguchi, H.; Gustafson, T. L. Ultrafast Time-Resolved Spontaneous and Coherent Raman Spectroscopy: The Structure and Dynamics of Photogenerated Transient Species. Annu. Rev. Phys. Chem. 1994, 45, 593-622.

(5) Nibbering, E. T. J.; Fidder, H.; Pines, E. Ultrafast chemistry: Using Time-Resolved Vibrational Spectroscopy for Interrogation of Structural Dynamics. Annu. Rev. Phys. Chem. 2005, 56, 337-367.

(6) Schmitt, M.; Knopp, G.; Materny, A.; Kiefer, W. Femtosecond time-resolved coherent anti-Stokes Raman scattering for the simultaneous study of ultrafast ground and excited state dynamics: iodine vapour. Chem. Phys. Lett. 1997, 270, 9-15.

(7) Kukura, P.; McCamant, D. W.; Mathies, R. A. Femtosecond stimulated Raman spectroscopy. Annu. Rev. Phys. Chem. 2007, 58, 461-488.

(8) Kukura, P.; McCamant, D. W.; Yoon, S.; Wandschneider, D. B.; Mathies, R. A. Structural observation of the primary isomerization in vision with femtosecondstimulated Raman. Science 2005, 310, 1006-1009.

(9) Prince, B. D.; Chakraborty, A.; Prince, B. M.; Stauffer, H. U. Development of simultaneous frequency- and time-resolved coherent anti-Stokes Raman scattering for ultrafast detection of molecular Raman spectra. J. Chem. Phys. 2006, 125, 044502.

(10) Atkinson, G. H.; Ujj, L.; Zhou, Y. Vibrational Spectrum of the J-625 Intermediate in the Room Temperature Bacteriorhodopsin Photocycle. J. Phys. Chem. A. 2000, 104, 41304139.

(11) Frontiera, R. R.; Shim, S.; Mathies, R. A. Origin of negative and dispersive features in anti-Stokes and resonance femtosecond stimulated Raman spectroscopy. J. Chem. Phys. 2008, 129, 064507.

(12) Ruhman, S.; Joly, A. G.; Nelson, K. A. Time-resolved observations of coherent molecular vibrational motion and the general occurrence of impulsive stimulated scattering. J. Chem. Phys. 1987, 86, 6563-6565.

(13) Fragnito, H. L.; Bigot, J. Y.; Becker, P. C.; Shank, C. V. Evoluition of the vibronic absorption-spectrum in a molecule following impulsive excitation with a 6 fs pulse. Chem. Phys. Lett. 1989, 160, 101-104.

(14) Ashworth, S. H.; Hasche, T.; Woerner, M.; Riedle, E.; Elsaesser, T. Vibronic excitations of large molecules in solution studied by two-color pump-probe experiments on the 20 fs time scale. J. Chem. Phys. 1996, 104, 5761.

(15) Dexheimer, S. L.; Wang, Q.; Peteanu, L. A.; Pollard, W. T.; Mathies, R. A.; Shank, C. V. Femtosecond impulsive excitation of nonstationary states in bacteriorhodopsin. Chem. Phys. Lett. 1992, 188, 61-66.

(16) Banin, U.; Bartana, A.; Ruhman, S.; Kosloff, R. Impulsive excitation of coherent vibrational motion ground surface dynamics induced by intense short pulses. J. Chem. 
Phys. 1994, 101, 8461-8481.

(17) Lanzani, G.; Cerullo, G.; Zavelani-Rossi, M.; De Silvestri, S. Sub-10 fs time resolved study of excited state relaxation in all-trans- $\beta$-carotene. Synthetic Met. 2001, 116, 1-3.

(18) Fujiyoshi, S.; Takeuchi, S.; Tahara, T. Time-Resolved Impulsive Stimulated Raman Scattering from Excited-State Polyatomic Molecules in Solution. J. Phys. Chem. A. 2003, 107, 494-500.

(19) Takeuchi, S.; Ruhman, S.; Tsuneda, T.; Chiba, M.; Taketsugu, T.; Tahara, T. Spectroscopic Tracking of Structural Evolution in Ultrafast Stilbene Photoisomerization. Science 2008, 322, 1073-1077.

(20) Kraack, J. P.; Buckup, T.; Motzkus, M. Coherent High-Frequency Vibrational Dynamics in the Excited Electronic State of All-Trans Retinal Derivatives. J. Phys. Chem. Lett. 2013, 4, 383-387.

(21) Hornung, T.; Skenderović, H.; Motzkus, M. Observation of all-trans- $\beta$-carotene wavepacket motion on the electronic ground and excited dark state using degenerate four-wave mixing (DFWM) and pump-DFWM. Chem. Phys. Lett. 2005, 402, 283-288.

(22) McCamant, D. W.; Kim, J. E.; Mathies, R. A. Vibrational Relaxation in $\beta$-Carotene Probed by Picosecond Stokes and Anti-Stokes Resonance Raman Spectroscopy. J. Phys. Chem. A. 2002, 106, 6030-6038.

(23) Shim, S.; Mathies, R. A. Development of a tunable femtosecond stimulated Raman apparatus and its application to beta-carotene. J. Phys. Chem. B 2008, 112, 4826-4832.

(24) Trautman, J. K.; Owens, T. G.; Albrecht, A. C. Determination of the S 2 lifetime of $\beta$ carotene. Chem. Phys. Lett. 1991, 178, 89-96.

(25) Perez Lustres, J. L.; Dobryakov, A. L.; Holzwarth, A.; Veiga, M. S2 -> S-1 internal conversion in beta-carotene: Strong vibronic coupling from amplitude oscillations of transient absorption bands. Angew. Chem. Int. Ed. 2007, 46, 3758-3761.

(26) Bradler, M.; Baum, P.; Riedle, E. Femtosecond continuum generation in bulk laser host materials with sub- $\mu \mathrm{J}$ pump pulses. Appl. Phys. B-Lasers $O . \mathbf{2 0 0 9}, 97,561-574$.

(27) Megerle, U.; Pugliesi, I.; Schriever, C.; Sailer, C. F.; Riedle, E. Sub-50 fs broadband absorption spectroscopy with tunable excitation: putting the analysis of ultrafast molecular dynamics on solid ground. Appl. Phys. B-Lasers O. 2009, 96, 215-231.

(28) Dobryakov, A. L.; Kovalenko, S. A.; Weigel, A.; Perez-Lustres, J. L.; Lange, J.; Muller, A.; Ernsting, N. P. Femtosecond pump/supercontinuum-probe spectroscopy: Optimized setup and signal analysis for single-shot spectral referencing. Rev. Sci. Instrum. 2010, $81,113106$.

(29) Pollard, W. T.; Mathies, R. A. Analysis ef femtosecond dynamics absorption spectra of nonstationary states. Annu. Rev. Phys. Chem. 1992, 43, 497-523.

(30) Kovalenko, S.; Dobryakov, A.; Ruthmann, J.; Ernsting, N. Femtosecond spectroscopy of condensed phases with chirped supercontinuum probing. Phys. Rev. A 1999, 59, 23692384.

(31) Polli, D.; Brida, D.; Mukamel, S.; Lanzani, G.; Cerullo, G. Effective temporal resolution in pump-probe spectroscopy with strongly chirped pulses. Phys. Rev. A 2010, 82, 053809.

(32) Dobryakov, A. L.; Kovalenko, S. A.; Ernsting, N. P. Coherent and sequential contributions to femtosecond transient absorption spectra of a rhodamine dye in solution. J. Chem. Phys. 2005, 123, 044502.

(33) Inagaki, F.; Tasumi, M.; Miyazawa, T. Excitation profile of the resonance Raman effect 
of $\beta$-carotene. J. Molec. Spectrosc. 1974, 50, 286-303.

(34) Kosumi, D.; Abe, K.; Karasawa, H.; Fujiwara, M.; Cogdell, R. J. Ultrafast relaxation kinetics of the dark S 1 state in all- trans- $\beta$-carotene explored by one-and two-photon pump-probe spectroscopy. Chem. Phys. 2010, 373, 33-37.

(35) McCamant, D.; Kukura, P.; Mathies, R. A. Femtosecond time-resolved stimulated Raman spectroscopy: Application to the ultrafast internal conversion in beta-carotene. $J$. Phys. Chem. A. 2003, 107, 8208-8214. 

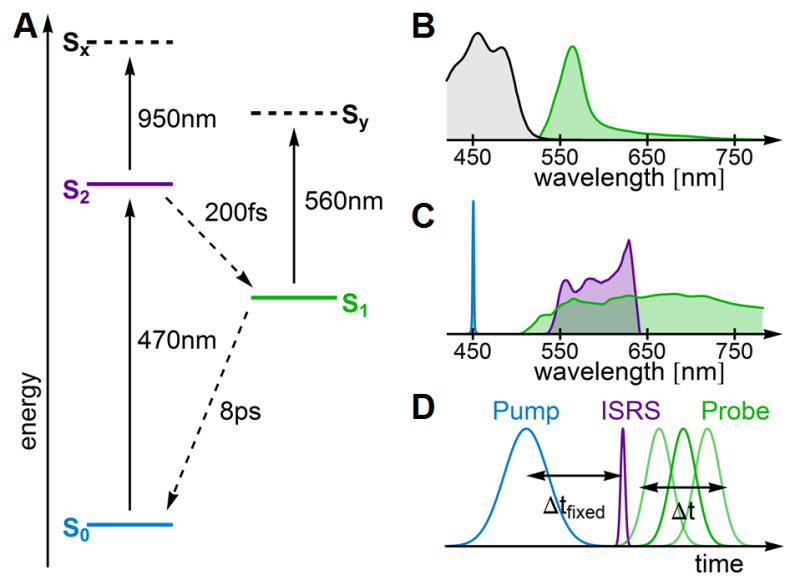

Figure 1. Broadband impulsive vibrational spectroscopy on $\beta$-carotene. (A) Commonly accepted energy level diagram for $\beta$-carotene. (B) Absorption spectra of the $S_{0}$ (black) and $S_{1}$ (green) states. (C) Spectra of the excitation pump (blue), impulsive pump (purple) and the probe pulse (green) used in broadband-IVS. (D) Corresponding relative pulse durations and timing. 

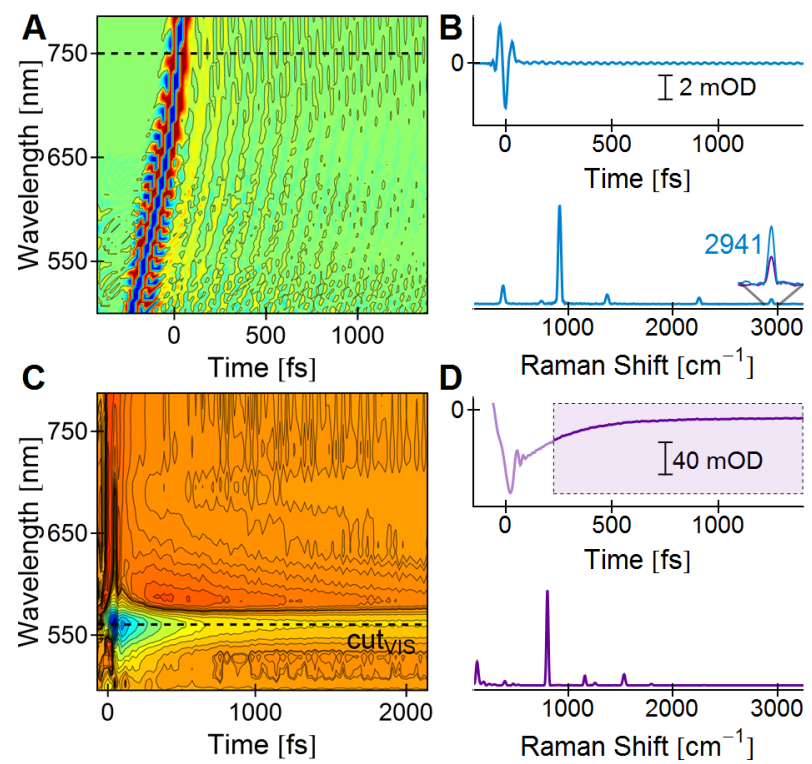

Figure 2. Probing high-frequency vibrational coherence in the time domain with chirped probe pulses. (A) Raw transient differential absorbance map of pure acetonitrile. The contrast of the oscillations in the visible is slightly reduced because of pump scattering. (B) Wavelength cut at $750 \mathrm{~nm}$ and corresponding Fourier power spectrum. The inset compares the $2941 \mathrm{~cm}-1$ peak at 750 (purple) and $560 \mathrm{~nm}$ (light blue). (C) Chirp corrected differential absorbance map of the $\mathrm{S}_{1}$ state of $\beta$-carotene using a $12 \mathrm{fs}$ impulsive pulse. (D) Differential absorbance cut at $560 \mathrm{~nm}$ and corresponding Fourier power spectrum after subtraction of the exponential kinetics in the boxed region. Data acquisition times for the maps in (A) and (C): 10 min. 


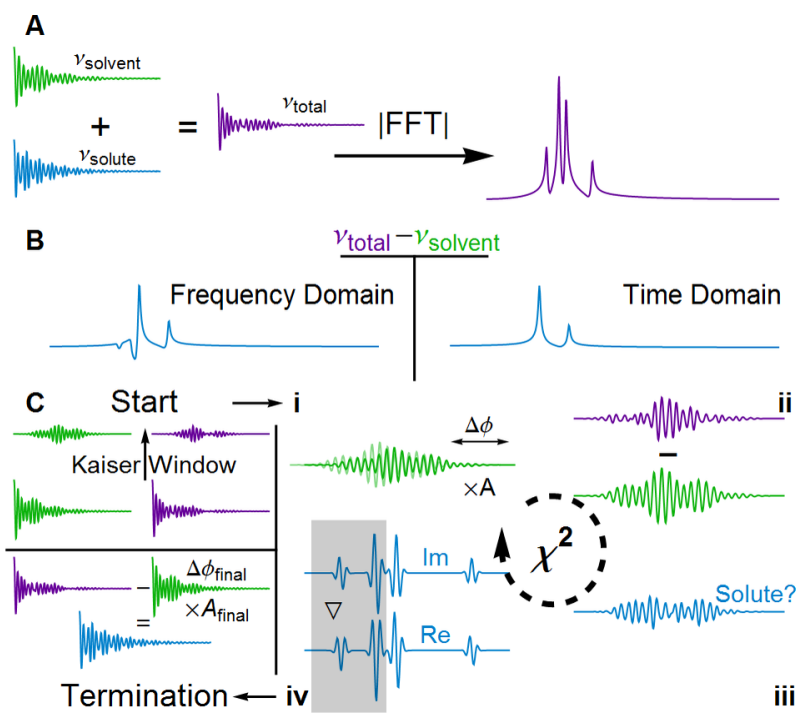

Figure 3. Subtraction of vibrational coherence in time and frequency domains. (A) Simulated vibrational coherence for a mixture of solvent (green) and solute (blue) in the time and frequency domain. (B) Subtraction of solvent features in the frequency domain causes dispersive lineshapes, while the subtraction in the time domain can be optimal. (C) Algorithm for optimised subtraction of background signatures in broadband impulsive vibrational spectroscopy. 

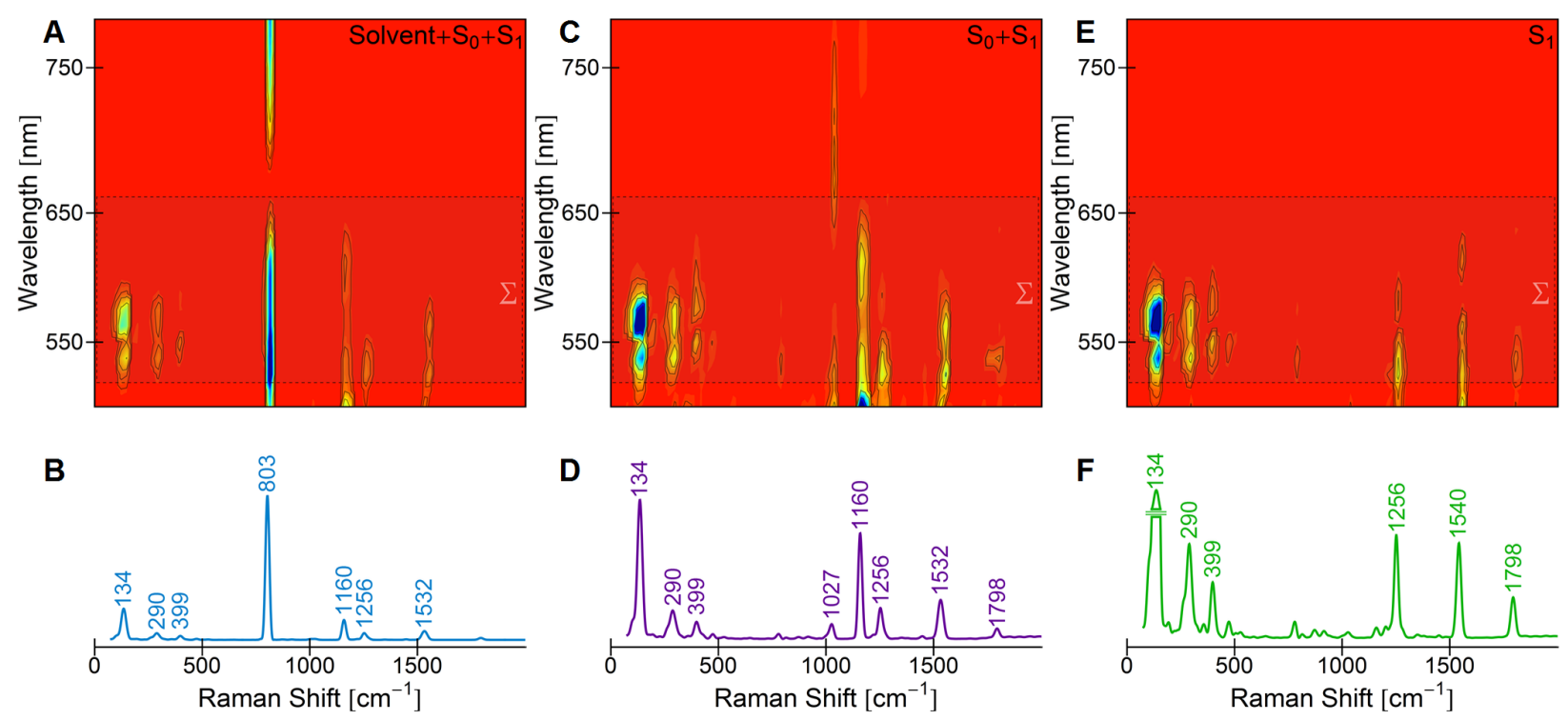

Figure 4. Fourier power maps and frequency spectra of coherent nuclear dynamics in $\beta$ carotene. (A) Wavelength resolved Fourier power map of $\beta$-carotene in cyclohexane in the presence of the UV excitation pulse. (B) Spectral average of the region indicated in (A). (C, D) Corresponding Fourier power map and spectrum after subtraction of the solvent signatures. (E, F) Pure $S_{1} \beta$-carotene map and spectrum after removal of ground state signatures. Acquisition time for all spectra: $10 \mathrm{~min}$. 

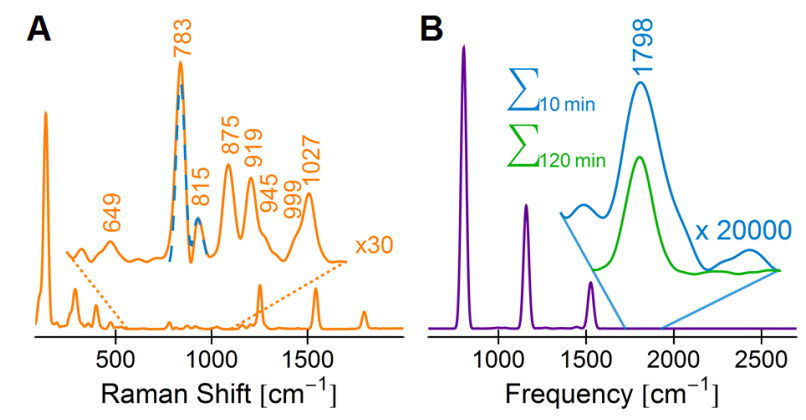

Figure 5. Sensitivity of broadband-IVS. (A) $S_{1}$-spectrum of $\beta$-carotene with a zoom of the 500 $1100 \mathrm{~cm}^{-1}$ region (inset). Dashed line: corresponding $\mathrm{S}_{1}$ spectrum from $760-830 \mathrm{~cm}^{-1}$ obtained in deuterated chloform. (B) Observation of high-frequency coherence in the $\mathrm{S}_{1}$ state generated by one-photon excitation in the absence of the UV excitation pulse. Inset: zoom of the $1800 \mathrm{~cm}^{-1}$ region for different integration times. 